\title{
SANDWICH MATRICES, SOLOMON ALGEBRAS, AND KAZHDAN-LUSZTIG POLYNOMIALS
}

\author{
MOHAN S. PUTCHA
}

\begin{abstract}
Sandwich matrices have proved to be of importance in semigroup theory for the last 50 years. The work of the author on algebraic monoids leads to sandwich matrices in group theory. In this paper, we find some connections between sandwich matrices and the Hecke algebras (for monoids) introduced recently by Louis Solomon. At the local level we then obtain an explicit isomorphism between Solomon's Hecke algebra and the complex monoid algebra of the Renner monoid. In the simplest case of monoids associated with a Borel subgroup, we find that the entries of the inverse of the sandwich matrix, as well as those of the related structure matrix of Solomon's Hecke algebra are 'almost' the polynomials $R_{x, y}$ associated with the Kazhdan-Lusztig polynomials.
\end{abstract}

\section{INTRODUCTION}

Modern semigroup theory essentially begins with the 1940 paper of Rees [21] giving the structure of a completely 0 -simple semigroup. Linear representation theory of semigroups begins with the 1942 paper of Clifford [2] on the irreducible representations of completely 0 -simple semigroups. Clifford's results became applicable to arbitrary finite semigroups via the work of Munn [9] and Ponizovskii [13]. In all these and subsequent works, the concept of a 'sandwich matrix' of a completely 0 -simple semigroup plays a critical role.

This paper is a contribution to the general program of the author to make semigroup or monoid representation theory relevant to group representation theory. Our primary focus will be on sandwich matrices of (finite) monoids $M$ of Lie type (such as $\mathscr{M}_{n}\left(\mathbb{F}_{q}\right)$ ). The local structure of $M$ is given by the 'local monoids' $M(J)=G \sqcup J \sqcup\{0\}$ where $G$ is the unit group and $J=G e G, e^{2}=e$. Such monoids can be constructed with respect to any parabolic subgroup $P=P_{I}$ of $G$ so that the local group at $e$ is the Levi subgroup $L$ of $P$. Semigroup representation theory then suggests the study of the sandwich matrix $S$ which is a $G / P^{-} \times G / P$ matrix over $\mathbb{C}[L]$. Passing to Solomon's Hecke algebra $\mathfrak{S}_{I}$ of $\mathbb{C}_{0}\left[J^{0}\right]$ yields a unipotent triangular matrix $\Omega$ over the Hecke algebra $H_{\mathbb{C}}(L)$ of $L$. We show that $\mathfrak{S}_{I}$ is naturally isomorphic to the Munn algebra over $H_{\mathbb{C}}(L)$ with sandwich matrix $\Omega$, whereby the inverse $\Omega^{-1}$ yields the unity of $\mathfrak{S}_{I}$. When $G$ is a Chevalley group over $\mathbb{F}_{q}$ and $P=B$ is a Borel subgroup, we show that the entries of $\Omega$ and $\Omega^{-1}$ are polynomials in $q$, closely related to the Kazhdan-Lusztig polynomials [8].

Received by the editors February 18, 1991 and, in revised form, September 23, 1991.

1991 Mathematics Subject Classification. Primary 20M30, 20G05.

Partially supported by NSF Grant DMS 9200077. 
We apply our results on local monoids to arbitrary finite monoids $M$ of Lie type. We give a direct proof that Solomon's Hecke algebra $\mathfrak{S}$ of $M$ is isomorphic to $\mathbb{C}_{0}[\mathfrak{R}]$ where $\mathfrak{R}$ is the Renner monoid of $M$. For $\mathscr{M}_{n}\left(\mathbb{F}_{q}\right)$, Solomon [24] proved this result by making use of the result of Okninski and the author [11] on the semisimplicity of $\mathbb{C}\left[\mathscr{M}_{n}\left(\mathbb{F}_{q}\right)\right]$.

\section{Preliminaries}

Let $M$ be a finite regular monoid (i.e., $a \in a M a$ for all $a \in M$ ) with zero 0 and group of units $G$. We are interested in the complex representation theory of $M$. By a representation of $M$ we will mean a homomorphism $\phi: M \rightarrow$ $\mathscr{M}_{n}(\mathbb{C})$ such that $\phi(1)=1$ and $\phi(0)=0$. Let $\mathbb{C}_{0}[M]$ denote the contracted semigroup algebra of $M$, i.e., the zero of $M$ is the zero of $\mathbb{C}_{0}[M]$. Then the representations of $M$ are in a natural 1-1 correspondence with the $\mathbb{C}_{0}[M]$ modules.

According to Green [6], the study of $M$ should begin with certain equivalence relations $\mathscr{J}, \mathscr{R}, \mathscr{L}, \mathscr{H}$, cf. [3]. For $a, b \in M, a \mathscr{J} b$ if $M a M=M b M$, $a \mathscr{R} b$ if $a M=b M, a \mathscr{L} b$ if $M a=M b, \mathscr{H}=\mathscr{R} \cap \mathscr{L}$. Let $\mathscr{U}=\mathscr{U}(M)$ denote the set of all $\mathscr{J}$-classes of $M$. Then $\mathscr{U}$ is a partially ordered set: $J_{1} \geq J_{2}$ if $J_{2} \subseteq M J_{1} M$. If $X \subseteq M$, we let $E(X)=\left\{e \in X \mid e^{2}=e\right\}$. Since $M$ is regular, $E(J) \neq \varnothing$ for all $J \in \mathscr{U}$. If $e \in E(M)$, then the $\mathscr{H}$-class $H$ of $e$ is just the group of units of $e M e$. We call $H$ the local group at $e$. Let $J \in \mathscr{U}$. We define

where for $a, b \in J$,

$$
M(J)=G \sqcup J \sqcup\{0\}
$$

$$
a \circ b= \begin{cases}a b & \text { if } a b \in J, \\ 0 & \text { otherwise. }\end{cases}
$$

Then $M(J)$ is a monoid which we call the local monoid at $J$. Let $J^{0}$ denote the subsemigroup $J \cup\{0\}$ of $M(J)$. Let $e \in E(J), H$ the local group at $e$. Let $R_{1}, \ldots, R_{n}$ and $L_{1}, \ldots, L_{m}$ denote the $\mathscr{R}$ and $\mathscr{L}$ classes of $J$, respectively with $H=R_{1} \cap L_{1}$. Choose $r_{i} \in R_{1} \cap L_{i}, i=1, \ldots, m, l_{j} \in L_{1} \cap R_{j}$, $j=1, \ldots, n$. Then $J^{0}, r_{i} l_{j} \in H \cup\{0\}, i=1, \ldots, m, j=1, \ldots, n$. The sandwich matrix $S$ is defined to be the matrix $\left(r_{i} l_{j}\right)$. Note that $S$ is not coordinate free, i.e. is dependent on the particular choice of $r_{i}, l_{j}$. Rees [21] showed that the completely 0 -simple semigroup $J^{0}$ is a matrix semigroup over $H \cup\{0\}$ with the multiplication twisted by $S$, cf. [3]. Clifford [2] showed that the irreducible representations of $J^{0}$ can be obtained from the irreducible representations $\phi$ of $H$ via a full rank factorization of $\phi(S)$ over $\mathbb{C}$. Munn [9] and Ponizovskii [13] showed that $\mathbb{C}_{0}\left[J^{0}\right]$ is semisimple if and only if $S$ is invertible over $\mathbb{C}[H]$, cf. [3]. In such a case $S^{-1}$ can be interpreted as the unity of $\mathbb{C}_{0}\left[J^{0}\right]$ (and in this sense coordinate free). If $S^{-1}=\left(a_{i j}\right)$, then the unity of $\mathbb{C}_{0}\left[J^{0}\right]$ is just $\sum l_{i} a_{i j} r_{j}$.

How is any of the above relevant to group representation theory? To establish a clear connection, we consider some special local monoids. The author [14-17] has shown that the local monoids of reductive monoids and finite monoids of Lie type (such as $\mathscr{M}_{n}(F)$ ) are all of this type. Let $G$ be a group and $P, P^{-}$ subgroups. Let $H$ be a group, $\theta: P \rightarrow H, \theta^{-}: P^{-} \rightarrow H$ homomorphisms such that $\left.\theta\right|_{P \cap P^{-}}=\left.\theta^{-}\right|_{P \cap P^{-}}$. Let

$$
M=M\left(G, P, P^{-}, H\right)=G \sqcup J \sqcup\{0\}, \quad J=(G \times H \times G) / \equiv,
$$


where for $a_{1}=\left(x_{1}, h_{1}, y_{1}\right), a_{2}=\left(x_{2}, h_{2}, y_{2}\right) \in G \times H \times G, a_{1} \equiv a_{2}$ if and only if $x_{2}^{-1} x_{1} \in P, y_{2} y_{1}^{-1} \in P^{-}, \theta\left(x_{2}^{-1} x_{1}\right) h_{1}=h_{2} \theta^{-}\left(y_{2} y_{1}^{-1}\right)$. If $a=(x, h, y)$, $b=\left(s, h^{\prime}, t\right) \in J, g \in G$, define

$$
\begin{array}{ll}
a b & = \begin{cases}\left(x, h \theta^{-}(q) \theta(p) h^{\prime}, t\right) & \text { if } y s=q p, q \in P^{-}, p \in P, \\
0 & \text { if } y s \notin P^{-} P,\end{cases} \\
a g & =(x, h, y g), \\
g a & =(g x, h, y) .
\end{array}
$$

Theorem 1.1. $M=M\left(G, P, P^{-}, H\right)$ is a monoid with the local group at $e=$ $(1,1,1)$ being isomorphic to $H$. Moreover as right and left $G$-modules,

$$
J / \mathscr{L} \cong G / P^{-}=\left\{P^{-} \alpha_{1}, \ldots, P^{-} \alpha_{m}\right\}, \quad J / \mathscr{R} \cong G / P=\left\{\beta_{1} P, \ldots, \beta_{n} P\right\},
$$

and the sandwich matrix $S=\left(\overline{\alpha_{i} \beta_{j}}\right)$ where for $g \in G$,

$$
\bar{g}= \begin{cases}\theta^{-}(q) \theta(p) & \text { if } g=q p, q \in P^{-}, p \in P, \\ 0 & \text { if } g \notin P^{-} P .\end{cases}
$$

Proof. It is clear that $\equiv$ is reflexive and symmetric. Let $a_{1}=\left(x_{1}, h_{1}, y_{1}\right)$, $a_{2}=\left(x_{2}, h_{2}, y_{2}\right), a_{3}=\left(x_{3}, h_{3}, y_{3}\right) \in G \times H \times G$ such that $a_{1} \equiv a_{2} \equiv a_{3}$. Then

$$
\begin{gathered}
x_{2}^{-1} x_{1}, x_{3}^{-1} x_{2} \in P, \quad y_{2} y_{1}^{-1}, y_{3} y_{2}^{-1} \in P^{-}, \\
\theta\left(x_{2}^{-1} x_{1}\right) h_{1}=h_{2} \theta^{-}\left(y_{2} y_{1}^{-1}\right), \quad \theta\left(x_{3}^{-1} x_{2}\right) h_{2}=h_{3} \theta^{-}\left(y_{3} y_{2}^{-1}\right) .
\end{gathered}
$$

Then $x_{3}^{-1} x_{1}=\left(x_{3}^{-1} x_{2}\right)\left(x_{2}^{-1} x_{1}\right) \in P, y_{3} y_{1}^{-1}=\left(y_{3} y_{2}^{-1}\right)\left(y_{2} y_{1}^{-1}\right) \in P^{-}$and

$$
\begin{aligned}
\theta\left(x_{3}^{-1} x_{1}\right) h_{1} & =\theta\left(x_{3}^{-1} x_{2}\right) \theta\left(x_{2}^{-1} x_{1}\right) h_{1}=\theta\left(x_{3}^{-1} x_{2}\right) h_{2} \theta^{-}\left(y_{2} y_{1}^{-1}\right) \\
& =h_{3} \theta^{-}\left(y_{3} y_{2}^{-1}\right) \theta^{-}\left(y_{2} y_{1}^{-1}\right)=h_{3} \theta^{-}\left(y_{3} y_{1}^{-1}\right) .
\end{aligned}
$$

Hence $a_{1} \equiv a_{3}$ and $\equiv$ is an equivalence relation on $G \times H \times G$. Next we show that the multiplication is well defined. So let $a=(x, h, y), a_{1}=\left(x_{1}, h_{1}, y_{1}\right)$, $b=\left(s, h^{\prime}, t\right), b_{1}=\left(s_{1}, h_{1}^{\prime}, t_{1}\right) \in G \times H \times G$ such that $a \equiv a_{1}$ and $b \equiv b_{1}$. Then

$$
\begin{gathered}
x_{1}^{-1} x, s_{1}^{-1} s \in P, \quad y_{1} y^{-1}, t_{1} t^{-1} \in P^{-}, \\
\theta\left(x_{1}^{-1} x\right) h=h_{1} \theta^{-}\left(y_{1} y^{-1}\right), \quad \theta\left(s_{1}^{-1} s\right) h^{\prime}=h_{1}^{\prime} \theta^{-}\left(t_{1} t^{-1}\right) .
\end{gathered}
$$

Suppose $a b \neq 0$. Then $y s \in P^{-} P$ and

$$
a b=\left(x, h \theta^{-}(q) \theta(p) h^{\prime}, t\right) \quad \text { where } y s=q p, q \in P^{-}, p \in P .
$$

Note that $\theta^{-}(q) \theta(p)$ is independent of the choice of $p$ and $q$ since $\left.\theta\right|_{P \cap P^{-}}=$ $\left.\theta^{-}\right|_{P \cap P^{-}}$. By (1),

$$
y_{1} s_{1}=\left(y_{1} y^{-1}\right)(y s)\left(s^{-1} s_{1}\right) \in P^{-}\left(P^{-} P\right) P=P^{-} P .
$$

By (1), $q_{1}=\left(y_{1} y^{-1}\right) q \in P^{-}$and $p_{1}=p\left(s^{-1} s_{1}\right) \in P$. Since $q p=y s, q_{1} p_{1}=$ $y_{1} s_{1}$. Hence

$$
a_{1} b_{1}=\left(x_{1}, h_{1} \theta^{-}\left(q_{1}\right) \theta\left(p_{1}\right) h_{1}^{\prime}, t_{1}\right) .
$$

By (1), $x_{1}^{-1} x \in P, t_{1} t^{-1} \in P^{-}$and

$$
\begin{aligned}
\theta\left(x_{1}^{-1} x\right) h \theta^{-}(q) \theta(p) h^{\prime} & =h_{1} \theta^{-}\left(y_{1} y^{-1}\right) \theta^{-}(q) \theta(p) h^{\prime}=h_{1} \theta^{-}\left(y_{1} y^{-1} q\right) \theta(p) h^{\prime} \\
& =h_{1} \theta^{-}\left(q_{1}\right) \theta(p) h^{\prime}=h_{1} \theta^{-}\left(q_{1}\right) \theta\left(p_{1}\left(s_{1}^{-1} s\right)\right) h^{\prime} \\
& =h_{1} \theta^{-}\left(q_{1}\right) \theta\left(p_{1}\right) \theta\left(s_{1}^{-1} s\right) h^{\prime}=h_{1} \theta^{-}\left(q_{1}\right) \theta\left(p_{1}\right) h_{1}^{\prime} \theta^{-}\left(t_{1} t^{-1}\right) .
\end{aligned}
$$


Hence $a b \equiv a_{1} b_{1}$. Similarly $a_{1} b_{1} \neq 0$ implies $a_{1} b_{1} \equiv a b$. It is also clear that for any $g \in G, g a \equiv g a_{1}$ and $a g \equiv a_{1} g$. It follows that the multiplication in $M$ is well defined.

We next prove associativity. So let $a, b, c \in M$. The only nontrivial case to consider is when $a, b, c \in J$. Let $a=\left(x_{1}, h_{1}, y_{1}\right), b=\left(x_{2}, h_{2}, y_{2}\right)$, $c=\left(x_{3}, h_{3}, y_{3}\right)$. Suppose $(a b) c \neq 0$. Then $a b \neq 0$ and $y_{1} x_{2} \in P^{-} P$. So

$$
a b=\left(x_{1}, h_{1} \theta^{-}(q) \theta(p) h_{2}, y_{2}\right) \quad \text { where } y_{1} x_{2}=q p, q \in P^{-}, p \in P \text {. }
$$

Since $(a b) c \neq 0, y_{2} x_{3} \in P^{-} P$. So

$$
\begin{array}{r}
(a b) c=\left(x_{1}, h_{1} \theta^{-}(q) \theta(p) h_{2} \theta^{-}\left(q^{\prime}\right) \theta\left(p^{\prime}\right) h_{3}, y_{3}\right) \\
\text { where } y_{2} x_{3}=q^{\prime} p^{\prime}, q^{\prime} \in P^{-}, p^{\prime} \in P .
\end{array}
$$

So

$$
b c=\left(x_{2}, h_{2} \theta^{-}\left(q^{\prime}\right) \theta\left(p^{\prime}\right) h_{3}, y_{3}\right)
$$

and $a(b c)=(a b) c$. Similarly $(a b) c \neq 0$ implies $a(b c)=(a b) c$. Hence $M$ is a monoid. Clearly the local group at $e$ is

$$
H^{\prime}=\{(1, h, 1) \mid h \in H\} \cong H .
$$

Let $a=(x, h, y) \in J$. Then $a=x(1, h, 1) y$. Hence $x e \mathscr{R} a \mathscr{L}$ ey. Let $y_{1}, y_{2} \in G$. Then $e y_{1} \mathscr{L} e y_{2}$ if and only if $e y_{1} y_{2}^{-1}=\left(1,1, y_{1} y_{2}^{-1}\right) \in H^{\prime}$. This happens exactly when $y_{1} y_{2}^{-1} \in P^{-}$, i.e., $P^{-} y_{1}=P^{-} y_{2}$. So

$$
J / \mathscr{L} \cong G / P^{-}=\left\{P^{-} \alpha_{1}, \ldots, P^{-} \alpha_{m}\right\} .
$$

Similarly

$$
J / \mathscr{R} \cong G / P=\left\{\beta_{1} P, \ldots, \beta_{n} P\right\}
$$

Clearly

$$
\left(e \alpha_{i}\right)\left(\beta_{j} e\right)=\left(1,1, \alpha_{i}\right)\left(\beta_{j}, 1,1\right)= \begin{cases}\left(1, \overline{\alpha_{i} \beta_{j}}, 1\right) & \text { if } \alpha_{i} \beta_{j} \in P^{-} P \\ 0 & \text { if } \alpha_{i} \beta_{j} \notin P^{-} P .\end{cases}
$$

This completes the proof of the theorem.

Remark 1.2. $J=G e G$ if and only if $H=\theta^{-}\left(P^{-}\right) \theta(P)$. This happens for example if $\theta$ or $\theta^{-}$is onto. In what we are interested in, both $\theta$ and $\theta^{-}$will be onto.

If $M$ is a monoid of Lie type, then by the author [16, 17], the local monoids of $M$ are of the form $M\left(G, P, P^{-}, L / K\right)$ where $P, P^{-}$are opposite parabolic subgroups, $L=P \cap P^{-}, K \triangleleft L$ and

$$
\theta: P=L U \rightarrow L / K, \quad \theta^{-}: P^{-}=L U^{-} \rightarrow L / K,
$$

the natural homomorphisms. If $K_{1} \triangleleft L, K \subseteq K_{1}$, then $M\left(G, P, P^{-}, L / K_{1}\right)$ is a natural homomorphic image of $M\left(G, P, P^{-}, L / K\right)$. Hence the universal monoid of this type is $M\left(G, P, P^{-}, L\right)$ and the smallest one is $M\left(G, P, P^{-}, 1\right)$. The sandwich matrix of $M\left(G, P, P^{-}, 1\right)$ is just the $G / P^{-} \times G / P$ matrix with the entry corresponding to $P^{-} \alpha, \beta P$ being 1 if $\alpha \beta \in P^{-} P$ and 0 if $\alpha \beta \notin$ $P^{-} P$. By the results of Okniński and the author [11], $\mathbb{C}[M]$ is semisimple for any monoid $M$ of Lie type. This result was proved by making use of HarishChandra's theory of cuspidal representations [1, Chapter 9]. For a theory of cuspidal representations of finite monoids, see [12]. By the results of Renner 
and the author [20] the irreducible modular representations of $M$ restrict to irreducible modular representations of $G$. In [20] a universal monoid $\mathscr{M}=$ $\mathscr{M}(G)$ of a particular type is constructed. The local monoids of $\mathscr{M}$ are exactly the various $M\left(G, P, P^{-}, L\right)$. The universal monoids of all possible types are constructed by the author [18].

\section{SANDWich Matrices and KaZHDAN-Lusztig POLYNOMIals}

Let $G$ be a finite group of Lie type with opposite Borel subgroups $B, B^{-}, T=$ $B \cap B^{-}$, and Weyl group $W=N / T$, cf. [1]. If $\sigma \in W$, let $\sigma=\dot{\sigma} T, \dot{\sigma} \in N$ with $\dot{T}=1$. Let $\ell(\sigma)$ denote the length of $\sigma$. Let $U, U^{-}$denote the unipotent radicals of $B, B^{-}$respectively, so that $B=T U$ and $B^{-}=T U^{-}$. We consider in this section the simplest local monoids, $M=M_{\varnothing}=M\left(G, B, B^{-}, T\right)$ and $M^{\prime}=M\left(G, B, B^{-}, 1\right)$ because the connection with the Kazhdan-Lusztig theory is most direct in this case. The general situation will be considered in the next section. In $\mathbb{C}[G]$, let

$$
\varepsilon=\frac{1}{|B|} \sum_{b \in B} b .
$$

Following Solomon [24] for $\mathscr{M}_{n}\left(\mathbb{F}_{q}\right)$, we consider the Hecke algebra

$$
\mathfrak{S}=\mathfrak{S}(M)=\varepsilon \mathbb{C}_{0}[M] \varepsilon .
$$

We will see shortly that

$$
\mathfrak{S}(M) \cong \mathfrak{S}\left(M^{\prime}\right)=\varepsilon \mathbb{C}_{0}\left[M^{\prime}\right] \varepsilon .
$$

Let

$$
\begin{aligned}
& M=G \sqcup J \sqcup\{0\}, \quad J=J_{\varnothing}=G e G, \quad e^{2}=e, \\
& M^{\prime}=G \sqcup J^{\prime} \sqcup\{0\}, \quad J^{\prime}=G e^{\prime} G, \quad e^{\prime 2}=e^{\prime} .
\end{aligned}
$$

Then $\mathbb{C}_{0}\left[J^{0}\right]$ is an ideal of $\mathbb{C}_{0}[M]$ and $\mathbb{C}[G] \cong \mathbb{C}_{0}[M] / \mathbb{C}_{0}\left[J^{0}\right]$. Hence

$$
\mathfrak{S}_{\varnothing}=\varepsilon \mathbb{C}_{0}\left[J^{0}\right] \varepsilon
$$

is an ideal of $\mathfrak{S}$ and

$$
\mathfrak{S} / \mathfrak{S}_{\varnothing} \cong \varepsilon \mathbb{C}[G] \varepsilon=H_{\mathbb{C}}(G)
$$

the Hecke algebra of $G$. Let $x, y \in G$. Then by the Bruhat and Birkhoff decompositions, $x \in B \sigma U, y \in U^{-} \theta B$ for some unique $\sigma, \theta \in W$. So $x e y \in B \sigma e \theta B$ and

$$
\varepsilon(x e y) \varepsilon=\varepsilon \dot{\sigma} e \dot{\theta} \varepsilon
$$

If $t \in T$, then

$$
\varepsilon(x t e y)=\varepsilon(x e y)
$$

and hence $\mathfrak{S}=\mathfrak{S}(M) \cong \mathfrak{S}\left(M^{\prime}\right)$. For $\sigma, \theta \in W$, let

$$
A_{\sigma, \theta}=\varepsilon \dot{\sigma}^{-1} e \dot{\theta} \varepsilon
$$

Then by (2), the $A_{\sigma, \theta}$ span $\mathfrak{S}_{\varnothing}$. Furthermore the supports of $A_{\sigma, \theta}$ and $A_{\sigma^{\prime}, \theta^{\prime}}$ have empty intersection unless $\sigma=\sigma^{\prime}$ and $\theta=\theta^{\prime}$. Hence $A_{\sigma, \theta}(\sigma, \theta \in W)$ form a basis for $\mathfrak{S}_{\varnothing}$. 
We will return to $\mathfrak{S}_{\varnothing}$ shortly. Let us consider the sandwich matrix of $J^{\prime 0} \subseteq$ $M^{\prime}$. For $\sigma \in W$, let

$$
\begin{aligned}
& V_{\sigma}=U \cap \dot{\sigma}^{-1} U \dot{\sigma}, \\
& Y_{\sigma}=U \cap \dot{\sigma}^{-1} U^{-} \dot{\sigma} .
\end{aligned}
$$

Then since $U$ is a product of its root subgroups in any order,

$$
V_{\sigma} \cap Y_{\sigma}=\{1\}, \quad U=V_{\sigma} Y_{\sigma}=Y_{\sigma} V_{\sigma} .
$$

Now $\dot{\sigma} v\left(v \in V_{\sigma}, \sigma \in W\right)$ is a complete set of distinct right coset representatives of $B^{-}$and $y \dot{\sigma}^{-1}\left(y \in Y_{\sigma}, \sigma \in W\right)$ is a complete set of distinct left coset representatives of $B$, cf. [11, proof of Lemma 2.5]. For $g \in G$, let

$$
\bar{g}= \begin{cases}1 & \text { if } g \in B^{-} B \\ 0 & \text { if } g \notin B^{-} B\end{cases}
$$

Then the sandwich matrix of $J^{\prime}$ is just the 0-1 matrix $S=\left(\overline{\dot{\theta} v y \dot{\sigma}^{-1}}\right)$. Moreover by [4] or [11, Lemma 2.2]

$$
\overline{\dot{\theta} v y \dot{\sigma}^{-1}}= \begin{cases}1 & \text { if } \theta=\sigma, \\ 0 & \text { if } \theta \not \leq \sigma,\end{cases}
$$

where $\leq$ denotes the usual Bruhat order on $W$, cf. [1, §1.9]. Thus if the elements of $W$ are arranged in length nondecreasing order (i.e., $W=\left\{\sigma_{1}, \sigma_{2}, \ldots\right\}$ with $\left.\ell\left(\sigma_{1}\right) \leq \ell\left(\sigma_{2}\right) \leq \cdots\right)$, then $S$ has a block upper triangular form with the blocks being rectangular. By [11] $S$ is invertible over $\mathbb{C}$. However $S^{-1}$ no longer has triangular form. In fact $S^{-1}$ has no zero entries at all! Amazingly, as we shortly see, the triangular form returns upon passing to the Solomon algebra. As pointed out before, $S^{-1}$ can be interpreted as the unity $h$ of $\mathbb{C}_{0}\left[J^{\prime}{ }^{0}\right]$. Let $a_{\theta}$ denote the $(1, \dot{\theta})$ entry of $S^{-1}$. Then $a_{\theta}$ is the coefficient of $e^{\prime} \dot{\theta}$ in $h$. Let $x, y \in G$ such that $y x \in B^{-} \sigma B$. Then $y x=b^{\prime} \dot{\sigma} b$ for some $b \in B$, $b^{\prime} \in B^{-}$. So

$$
b x^{-1}\left(x e^{\prime} y\right) x b^{-1}=b e^{\prime} y x b^{-1}=b e^{\prime} b^{\prime} \dot{\sigma}=e^{\prime} \dot{\sigma} .
$$

So $x e^{\prime} y$ and $e^{\prime} \dot{\sigma}$ are conjugate. Since $h$ is central in $\mathbb{C}_{0}\left[M^{\prime}\right]$, we see that $a_{\sigma}$ is also the coefficient of $x e^{\prime} y$ in $h$. Thus if

$$
L_{\sigma}=\sum\left\{x e y \mid x, y \in G, y x \in B^{-} \sigma B\right\}
$$

then we have,

Proposition 2.1. The unity $h$ of $\mathbb{C}_{0}\left[J^{\prime}\right]$ is given by

$$
h=\sum_{\sigma \in W} a_{\sigma} L_{\sigma}
$$

We wish to compute the numbers $a_{\sigma}$. For $\sigma, \theta \in W$, let

$$
Q_{\theta, \sigma}=\frac{\left|\dot{\theta} B \dot{\sigma}^{-1} \cap B^{-} B\right|}{|B|}=\frac{\left|\dot{\theta} U \dot{\sigma}^{-1} \cap B^{-} B\right|}{|U|} .
$$

Then

$$
\frac{\left|\dot{\theta} U \dot{\sigma}^{-1} \cap B^{-} B\right|}{\left|Y_{\theta}\right|}=\frac{Q_{\theta, \sigma} \cdot|U|}{\left|Y_{\theta}\right|}=Q_{\theta, \sigma} \cdot\left|V_{\theta}\right| .
$$


By [11, equations (17), (18)], the numbers $a_{\sigma}$ satisfy the following system of equations:

$$
\begin{aligned}
|U| a_{1} & =1, \\
\sum_{\theta \in W}\left|V_{\theta}\right| \cdot Q_{\theta, \sigma} a_{\theta} & =0 \quad \text { for } \sigma \neq 1 .
\end{aligned}
$$

We are therefore led to considering the matrix

$$
\Omega_{\varnothing}=\left(Q_{\theta, \sigma}\right) \text {. }
$$

We call $\Omega_{\varnothing}$ the structure matrix of $\mathfrak{S}_{\varnothing}$. Now by [4] or [11, Lemma 2.2]

$$
Q_{\theta, \sigma}= \begin{cases}1 & \text { if } \theta=\sigma, \\ 0 & \text { if } \theta \not \leq \sigma,\end{cases}
$$

where $\leq$ denotes the Bruhat order on $W$. Thus if the elements of $W$ are listed in length nondecreasing order, then $\Omega_{\phi}$ is an upper triangular unipotent matrix and is in particular invertible. Let

$$
\Omega_{\varnothing}^{-1}=\left(\tilde{Q}_{\theta, \sigma}\right) \text {. }
$$

Then by (8), we have

Proposition 2.2. For all $\sigma \in W, a_{\sigma}=\widetilde{Q}_{1, \sigma} /\left|V_{\sigma}\right|$.

We will show that $Q_{\theta, \sigma}$ and $\widetilde{Q}_{\theta, \sigma}$ are related to some polynomials in Kazhdan-Lusztig theory. Before doing this we use $\Omega_{\varnothing}$ and $\Omega_{\varnothing}^{-1}$ to study the structure of the Solomon algebra $\mathfrak{S}_{\varnothing}$. Let $x \in \mathfrak{S}_{\varnothing}$. Then $x=\sum_{\sigma, \theta \in W} \alpha_{\sigma, \theta} A_{\sigma, \theta}$ for some unique $\alpha_{\sigma, \theta} \in \mathbb{C}$. Define $\phi(x)=\left(\alpha_{\sigma, \theta}\right) \in \mathscr{M}_{n}(\mathbb{C})$ where $n=|W|$.

Theorem 2.3. The map $\psi: \mathfrak{S}_{\varnothing} \rightarrow \mathscr{M}_{n}(\mathbb{C})$ given by $\psi(x)=\phi(x) \cdot \Omega$, where $\Omega=\Omega_{\varnothing}$ is the structure matrix of $\mathfrak{S}_{\varnothing}$, is an algebra isomorphism. In particular the unity $h^{\prime}$ of $\mathfrak{S}_{\varnothing}$ is given by

$$
h^{\prime}=\varepsilon h=\sum_{\theta, \sigma \in W} \widetilde{Q}_{\theta, \sigma} \cdot A_{\theta, \sigma} .
$$

Proof. We have already seen that $\phi$ and hence $\psi$ (since $\Omega$ is invertible) is a vector space isomorphism. It suffices therefore to show that for all $x, y \in \mathfrak{S}_{\varnothing}$,

$$
\phi(x y)=\phi(x) \Omega \phi(y) \text {. }
$$

By linearity, it suffices to consider the case when $x=A_{\sigma, \theta}=\varepsilon \dot{\sigma}^{-1} e \dot{\theta} \varepsilon, y=$ $A_{\sigma^{\prime}, \theta^{\prime}}=\varepsilon \dot{\sigma}^{\prime-1} e \dot{\theta}^{\prime} \varepsilon$. Let $b \in B$. If $\dot{\theta} b \dot{\sigma}^{\prime-1} \notin B^{-} B$, then $e \dot{\theta} b \dot{\sigma}^{\prime-1} e=0$. Suppose $\dot{\theta} b \dot{\sigma}^{-1} \in B^{-} B$. Then $\dot{\theta} b \dot{\sigma}^{-1} \in U^{-} t U$ for some $t \in T$ and $e \dot{\theta} b \dot{\sigma}^{-1} e=$ te. Thus by (3)

$$
x y=\frac{\left|\dot{\theta} B \dot{\sigma}^{\prime-1} \cap B^{-} B\right|}{|B|} \cdot \varepsilon \dot{\sigma}^{-1} e \dot{\theta}^{\prime} \varepsilon=Q_{\theta, \sigma^{\prime}} A_{\sigma, \theta^{\prime}} .
$$

Hence $\phi(x y)=\phi(x) \Omega \phi(y)$, establishing (10). In particular $1=\psi\left(h^{\prime}\right)=\phi\left(h^{\prime}\right) \Omega$ and $\phi\left(h^{\prime}\right)=\Omega^{-1}$. This completes the proof.

Kazhdan and Lusztig [8] constructed the now famous polynomials $P_{\theta, \sigma}(q)$ $(\theta, \sigma \in W)$. They also consider some closely related polynomials, $R_{\theta, \sigma}(q)$ $(\theta, \sigma \in W)$. Deodhar [4, 5] further studied these polynomials and in particular found a closed form formula for $R_{\theta, \sigma}(q)$. 
Theorem 2.4. Suppose $G$ is a Chevalley group over $\mathbb{F}_{q}$ and let $m$ denote the number of positive roots. Then

(i) $Q_{\theta, \sigma}=q^{\ell(\theta)-\ell(\sigma)} R_{\theta, \sigma}(q)$ for all $\theta, \sigma \in W$.

(ii) $\widetilde{Q}_{\theta, \sigma}=(-q)^{\ell(\theta)-\ell(\sigma)} R_{\theta, \sigma}(q)$ for all $\theta, \sigma \in W$.

(iii) For all $\sigma \in W, a_{\sigma}=q^{-m}(-1)^{\ell(\sigma)} R_{1, \sigma}(q)$.

(iv) The unity $h$ of $\mathbb{C}_{0}\left[J^{\prime 0}\right]$ is given by

$$
h=q^{-m} \sum_{\sigma \in W}(-1)^{\ell(\sigma)} R_{1, \sigma}(q) L_{\sigma} .
$$

(v) The unity $h^{\prime}$ of $\mathfrak{S}_{\varnothing}$ is given by

$$
h^{\prime}=\varepsilon h=\sum_{\theta, \sigma \in W}(-q)^{\ell(\theta)-\ell(\sigma)} R_{\theta, \sigma}(q) A_{\theta, \sigma} .
$$

Proof. By (6), [1, Proposition 2.2.7] we see that

$$
|U|=q^{m}, \quad\left|Y_{\sigma}\right|=q^{\ell(\sigma)}, \quad\left|V_{\sigma}\right|=q^{m-\ell(\sigma)} .
$$

By $[4,8]$,

$$
R_{\theta, \sigma}(q)=\left|B^{-} \theta \cdot B \cap B \sigma \cdot B\right|
$$

in $G / B$. Hence in $G$,

$$
R_{\theta, \sigma}(q)=\left|B^{-} \theta B \cap B \sigma B\right| /|B| .
$$

By (4), (5), (6), (11) we see that

$$
\left|\dot{\theta} U \dot{\sigma}^{-1} \cap B^{-} B\right|=\left|Y_{\theta}\right| \cdot\left|\dot{\theta} V_{\theta} \dot{\sigma}^{-1} \cap B^{-} B\right|=q^{\ell(\theta)}\left|\dot{\theta} V_{\theta} \dot{\sigma}^{-1} \cap B^{-} B\right| .
$$

Let $v \in V_{\theta}$ such that $\dot{\theta} v \dot{\sigma}^{-1} \in B^{-} B=B^{-} U$. Then $\dot{\theta} v \dot{\sigma}^{-1}=b u$ for unique $b \in B^{-}, u \in U$ and

$$
u \dot{\sigma}=b^{-1} \dot{\theta} v \in B^{-} \theta B .
$$

Conversely let $u \in U$ such that $u \dot{\sigma} \in B^{-} \theta B=B^{-} \dot{\theta} V_{\theta}$. Then $u \dot{\sigma}=b^{-1} \dot{\theta} v$ for some unique $b \in B^{-}$and $v \in V_{\theta}$. So

$$
\dot{\theta} v \dot{\sigma}^{-1}=b u \in B^{-} B \text {. }
$$

Hence

$$
\left|\dot{\theta} V_{\theta} \dot{\sigma}^{-1} \cap B^{-} B\right|=\left|U \dot{\sigma} \cap B^{-} \theta B\right| .
$$

By (4), (5), (6),

$$
\left|U \dot{\sigma} \cap B^{-} \theta B\right|=\left|V_{\sigma^{-1}}\right| \cdot\left|Y_{\sigma^{-1}} \dot{\sigma} \cap B^{-} \theta B\right| .
$$

Since $B \sigma B=Y_{\sigma^{-1}} \sigma B$, we see by (12) or (13) that

$$
\left|Y_{\sigma^{-1}} \dot{\sigma} \cap B^{-} \theta B\right|=R_{\theta, \sigma}(q) \text {. }
$$

Since $\ell(\sigma)=\ell\left(\sigma^{-1}\right)$, we see by (7), (11), (14)-(17) that $Q_{\theta, \sigma}=q^{\ell(\theta)-\ell(\sigma)} R_{\theta, \sigma}(q)$. By [8, proof of Lemma 2.1], the matrices

$$
A_{1}=\left(q^{\ell(\theta)} R_{\theta, \sigma}\right) \text { and } A_{2}=\left((-1)^{\ell(\theta)}(-q)^{-\ell(\sigma)} R_{\theta, \sigma}\right)
$$

are inverses. Let $D$ be the diagonal matrix $\operatorname{diag}\left(q^{-\ell(\sigma)}\right)$. Then $\Omega_{\varnothing}=A_{1} D$ and

$$
\Omega_{\varnothing}^{-1}=D^{-1} A_{2}=\left((-q)^{\ell(\theta)-\ell(\sigma)} R_{\theta, \sigma}\right) .
$$


This yields (ii) by (9). (iii), (iv), (v) now follow from (11), Propositions 2.1, 2.2, and Theorem 2.3.

Problem 2.5. If $G$ is a Chevalley group, then by Theorem 2.4, $\widetilde{Q}_{\theta, \sigma}=$ $(-1)^{\ell(\theta)-\ell(\sigma)} Q_{\theta, \sigma}$. Is this valid for any group of Lie type?

\section{LOCAL MONOIDS}

We begin this section by considering universal local monoids. We will then apply these results to arbitrary monoids of Lie type in the next section. Let $G, B, B^{-}, N, T, U, U^{-}, W$ be as in the previous section. Let $\Gamma$ denote the Coxeter graph of $G$. Fix a subset $I$ of $\Gamma$. Let $P=P_{I}=B W_{I} B, P^{-}=P_{I}^{-}=$ $B^{-} W_{I} B^{-}, L=L_{I}=P \cap P^{-}, U_{I}$ the unipotent radical of $P$, and $U_{I}^{-}$the unipotent radical of $P^{-}$. Let

$$
M_{I}=M\left(G, P, P^{-}, L\right)=G \sqcup J_{I} \sqcup\{0\}, \quad J_{I}=G e_{I} G, e_{I}^{2}=e_{I} .
$$

Then

$$
U_{I} e_{I}=\left\{e_{I}\right\}=e_{I} U_{I}^{-}, \quad L e_{I}=e_{I} L
$$

Let

$$
\varepsilon=\frac{1}{|B|} \sum_{b \in B} b, \quad \mathfrak{S}_{I}=\varepsilon \mathbb{C}_{0}\left[J_{I}\right] \varepsilon .
$$

We are interested in the structure of $\mathfrak{S}_{I}$. Let

$$
B_{L}=B \cap L, \quad \varepsilon_{I}=\frac{1}{\left|B_{L}\right|} \sum_{b \in B_{L}} b
$$

and

$$
H_{\mathbb{C}}(L)=\varepsilon_{I} \mathbb{C}[L] \varepsilon_{I}
$$

the Hecke algebra of $L$. Then $\varepsilon_{I}$ is the unity of $H_{\mathbb{C}}(L)$. For $\pi \in W_{I}$, let

$$
T_{\pi}^{\prime}=\varepsilon_{I} \dot{\pi} \varepsilon_{I} \in H_{\mathbb{C}}(L) .
$$

These are scalar multiples of the usual basis elements $T_{\pi}$ of Iwahori [7]. By a theorem of Tits, cf. $[1, \S 10,11], H_{\mathbb{C}}(L) \cong \mathbb{C}\left[W_{I}\right]$. Let

$$
D_{I}=\left\{\sigma \in W \mid \ell(\sigma \theta) \geq \ell(\sigma) \text { for all } \theta \in W_{I}\right\}, \quad D_{I}^{-1}=\left\{\sigma^{-1} \mid \sigma \in D_{I}\right\} .
$$

Then $D_{I}$ is a complete set of distinct left coset representatives of $W_{I}$ and $D_{I}^{-1}$ is a complete set of distinct right coset representatives of $W_{I}$. By [1, Propositions 2.3.3, 2.5.15] we see that for $\sigma \in W$,

$$
\sigma \in D_{I} \text { if and only if } \dot{\sigma} B_{L} \dot{\sigma}^{-1} \subseteq B .
$$

Since $U$ is a product of its root subgroups in any order, we see that

$$
\dot{\sigma}^{-1} B \dot{\sigma} \subseteq U_{I}^{-} B \text { for all } \sigma \in D_{I} .
$$

If $\left|D_{I}\right|=n$, we will exhibit an explicit isomorphism between $\mathfrak{S}_{I}$ and $\mathscr{M}_{n}\left(H_{\mathbb{C}}(L)\right)$. By [11, proof of Lemma 2.5],

$$
G=\bigsqcup_{\sigma \in D_{I}} B \sigma P=\bigsqcup_{\sigma \in D_{I}} P^{-} \sigma^{-1} B
$$


Let $a \in J_{I}^{0}$. Then $a=x e_{I} y$ for some $x, y \in G$. By (21), there exist $\sigma, \theta \in D_{I}$ such that $x \in B \sigma P, y \in P^{-} \theta^{-1} B$. So by (18),

$$
a=x e_{I} y \in B \sigma P e_{I} P^{-} \theta^{-1} B=B \sigma L e_{I} \theta^{-1} B .
$$

Hence for some $\pi \in W_{I}$,

$$
a \in B \sigma B_{L} \pi B_{L} e_{I} \theta^{-1} B=B \sigma \pi e_{I} \theta^{-1} B, \quad \text { by (19). }
$$

We claim that $\sigma, \pi, \theta$ are unique. For suppose for some $\sigma_{1}, \theta_{1} \in D_{I}, \pi_{1} \in$ $W_{I}, a \in B \sigma_{1} \pi_{1} e_{I} \theta_{1}^{-1} B$. Then

$$
B \sigma_{1} P=B \sigma_{1} \pi_{1} P=B \sigma \pi P=B \sigma P, \quad P^{-} \theta_{1}^{-1} B=P^{-} \theta^{-1} B .
$$

Hence by (21), $\sigma=\sigma_{1}, \theta=\theta_{1}$. Since $\pi \in W_{I}$,

$$
\begin{aligned}
\dot{\sigma}^{-1} a \dot{\theta} & \in \sigma^{-1} B \sigma \pi e_{I} \theta^{-1} B \theta \cap \sigma^{-1} B \sigma \pi_{1} e_{I} \theta^{-1} B \theta \\
& \subseteq U_{I}^{-} B \pi e_{I} U_{I}^{-} B \cap U_{I}^{-} B \pi_{1} e_{I} U_{I}^{-} B \text { by }(20) \\
& =U_{I}^{-} B_{L} \pi e_{I} B \cap U_{I}^{-} B_{L} \pi_{1} e_{I} B \text { by }(18) .
\end{aligned}
$$

So by (18),

$$
e_{I} \dot{\sigma}^{-1} a \dot{\theta} e_{I} \in B_{L} \pi B_{L} e_{I} \cap B_{L} \pi_{1} B_{L} e_{I} .
$$

Hence $B_{L} \pi B_{L} \cap B_{L} \pi_{1} B \neq \varnothing$ and $\pi=\pi_{1}$. Thus $\sigma, \pi, \theta$ are unique. Also

$$
\varepsilon a \varepsilon=\varepsilon \dot{\sigma} \dot{\pi} e_{I} \dot{\theta}^{-1} \varepsilon=\varepsilon \dot{\sigma} T_{\pi}^{\prime} e_{I} \dot{\theta}^{-1} \varepsilon \text { by (18), (19). }
$$

For $\sigma, \theta \in D_{I}^{-1}, \pi \in W_{I}$, define a linear transformation $A_{\sigma, \theta}: H_{\mathbb{C}}(L) \rightarrow \mathfrak{S}_{I}$ as

$$
A_{\sigma, \theta}\left(T_{\pi}^{\prime}\right)=\varepsilon \dot{\sigma}^{-1} \dot{\pi} e_{I} \dot{\theta} \varepsilon=\varepsilon \dot{\sigma}^{1} T_{\pi}^{\prime} e_{I} \dot{\theta} \varepsilon .
$$

By the preceding discussion $A_{\sigma, \theta}\left(T_{\pi}^{\prime}\right)$ with $\sigma, \theta \in D_{I}^{-1}, \pi \in W_{I}$ span $\mathfrak{S}_{I}$ and have pairwise disjoint support. Hence they form a basis for $\mathfrak{S}_{I}$. For $\sigma, \theta \in D_{I}^{-}$, let

$$
Q_{\theta, \sigma}^{I}=\sum_{\pi \in W_{I}} \frac{\left|\dot{\theta} B \dot{\sigma}^{-1} \cap U_{I}^{-} B_{L} \pi B\right|}{|B|} \cdot T_{\pi}^{\prime} \in H_{\mathbb{C}}(L) .
$$

By (20),

$$
Q_{\sigma, \sigma}^{I}=\varepsilon_{I} \quad \text { for all } \sigma \in D_{I}^{-1} .
$$

If $\theta \not \leq \sigma$, then by [11, proof of Lemma 2.2], $\theta B \cap P^{-} P \sigma=\varnothing$ and hence

$$
Q_{\theta, \sigma}^{I}=0 \text { if } \theta, \sigma \in D_{I}^{-1}, \theta \leq \sigma,
$$

where $\leq$ denotes the Bruhat order on $W$. We define the structure matrix $\Omega_{I}$ of $\mathfrak{S}_{I}$ as

$$
\Omega_{I}=\left(Q_{\theta, \sigma}^{I}\right) \in \mathscr{M}_{n}\left(H_{\mathbb{C}}(L)\right) \text { where } n=\left|D_{I}\right| \text {. }
$$

If we arrange the elements of $D_{I}^{-1}$ in length nondecreasing order, then $\Omega_{I}$ is an upper triangular unipotent matrix. In particular $\Omega_{I}$ is invertible. Let $\Omega_{I}^{-1}=$ $\left(\widetilde{Q}_{\theta, \sigma}^{I}\right)$. Let $x \in \mathfrak{S}_{I}$. Then $x=\sum_{\theta, \sigma \in D_{I}^{-1}} A_{\sigma, \theta}\left(\pi_{\sigma, \theta}\right)$ for some unique $\pi_{\sigma, \theta} \in$ $H_{\mathbb{C}}(L)$. Define $\phi(x)=\left(\pi_{\sigma, \theta}\right) \in \mathscr{M}_{n}\left(H_{\mathbb{C}}(L)\right)$. Then we have the following generalization of Theorem 2.3. 
Theorem 3.1. The map $\psi: \mathfrak{S}_{I} \rightarrow \mathscr{M}_{n}\left(H_{\mathbb{C}}(L)\right)$ given by $\psi(x)=\phi(x) \cdot \Omega$, where $\Omega=\Omega_{I}$ is the structure matrix of $\mathfrak{S}_{I}$, is an algebra isomorphism. In particular the unity $h_{I}$ of $\mathfrak{S}_{I}$ is given by

$$
h_{I}=\sum_{\theta, \sigma \in D_{I}^{-1}} A_{\theta, \sigma}\left(\tilde{Q}_{\theta, \sigma}^{I}\right) .
$$

Proof. We have already seen that $\phi$ and $\psi$ (since $\Omega$ is invertible) is a vector space isomorphism. It suffices therefore to show that for all $x, y \in \mathfrak{S}_{I}$,

$$
\phi(x y)=\phi(x) \Omega \phi(y) \text {. }
$$

By linearity, it suffices to consider the case when

$$
\begin{aligned}
& x=A_{\sigma, \theta}\left(T_{\pi}^{\prime}\right)=\varepsilon \dot{\sigma}^{-1} T_{\pi}^{\prime} e_{I} \dot{\theta} \varepsilon, \\
& y=A_{\sigma^{\prime}, \theta^{\prime}}\left(T_{\pi^{\prime}}^{\prime}\right)=\varepsilon \dot{\sigma}^{\prime-1} T_{\pi^{\prime}}^{\prime} e_{I} \dot{\theta}^{\prime} \varepsilon=\varepsilon \dot{\sigma}^{\prime-1} e_{I} T_{\pi^{\prime}}^{\prime} \dot{\theta}^{\prime} \varepsilon .
\end{aligned}
$$

Let $b \in B$. If $\dot{\theta} b \dot{\sigma}^{-1} \notin P-P$, then $e_{I} \dot{\theta} b \dot{\sigma}^{-1} e_{I}=0$. Now

$$
P^{-} P=U_{I}^{-} L U_{I}=\bigsqcup_{\gamma \in W_{I}} U_{I}^{-} B_{L} \gamma B_{L} U_{I}=\bigsqcup_{\gamma \in W_{I}} U_{I}^{-} B_{L} \gamma B .
$$

If $\dot{\theta} b \dot{\sigma}^{\prime-1} \in U_{I}^{-} B_{L} \gamma B_{L} U_{I}$, then

$$
T_{\pi}^{\prime} e_{I} \dot{\theta} b \dot{\sigma}^{\prime-1} e_{I} T_{\pi^{\prime}}^{\prime}=e_{I} T_{\pi}^{\prime} T_{\gamma}^{\prime} T_{\pi^{\prime}}^{\prime}=T_{\pi}^{\prime} T_{\gamma}^{\prime} T_{\pi^{\prime}}^{\prime} e_{I}
$$

Hence

$$
\begin{aligned}
x y & =\sum_{\gamma \in W_{I}} \frac{\left|\dot{\theta} B \dot{\sigma}^{\prime-1} \cap U_{I}^{-} B_{L} \gamma B\right|}{|B|} \cdot \varepsilon \dot{\sigma}^{\prime-1} T_{\pi}^{\prime} T_{\gamma}^{\prime} T_{\pi^{\prime}}^{\prime} e_{I} \dot{\theta}^{\prime} \varepsilon \\
& =\varepsilon \dot{\sigma}^{-1} T_{\pi}^{\prime} Q_{\theta, \sigma^{\prime}}^{I} T_{\pi^{\prime}}^{\prime} e_{I} \dot{\theta}^{\prime} \varepsilon=A_{\sigma, \theta^{\prime}}\left(T_{\pi}^{\prime} Q_{\theta, \sigma^{\prime}}^{I} T_{\pi^{\prime}}^{\prime}\right) .
\end{aligned}
$$

Hence $\phi(x y)=\phi(x) \Omega \phi(y)$, establishing (22). In particular $1=\psi\left(h_{I}\right)=$ $\phi\left(h_{I}\right) \Omega$ and $\phi\left(h_{I}\right)=\Omega^{-1}$. This completes the proof.

Remark 3.2. In the terminology of $[3, \S 5.2], \mathfrak{S}_{I}$ is a Munn algebra over $H_{\mathbb{C}}(L)$ with sandwich matrix $\Omega_{I}$.

Problem 3.3. The elements $\widetilde{Q}_{\theta, \sigma}^{I}$ of the Hecke algebra $H_{\mathbb{C}}(L)$ can be computed recursively via the Bruhat order. Can a closed form expression be found?

Let us now consider the general local monoid $M^{\prime}=\left(G, P, P^{-}, L^{\prime}\right)$ where $L^{\prime}=L / K, K \triangleleft L$. Let $J^{\prime}=G e_{I} G$ in $M^{\prime}$. Consider the Hecke algebra $\mathfrak{S}_{I}^{\prime}=\varepsilon \mathbb{C}_{0}\left[J^{\prime 0}\right] \varepsilon$. Let $f: \mathbb{C}[L] \rightarrow \mathbb{C}\left[L^{\prime}\right]$ denote the natural homomorphism. Let

$$
I^{\prime}=\left\{\pi \in W_{I} \mid f(\dot{\pi}) \notin f\left(B_{L}\right)\right\} .
$$

Then by [1, Proposition 2.1.5], $W_{I^{\prime}}$ is the Weyl group of $L^{\prime}$ with respect to $f\left(B_{L}\right), f(N \cap L)$. So if $T_{\pi}\left(\pi \in W_{I}\right)$ is the usual basis of $H_{\mathbb{C}}(L)$, cf. [1, Chapter 10], then $f\left(T_{\pi}\right)\left(\pi \in W_{I^{\prime}}\right)$ is a basis for $f\left(H_{\mathbb{C}}(L)\right)$. Hence by Tits' theorem, cf. [1, $\S 10.11], f\left(H_{\mathbb{C}}(L)\right) \cong \mathbb{C}\left[W_{I^{\prime}}\right]$. So by Theorem 3.1 we have

Corollary 3.4. $\mathfrak{S}_{I}^{\prime} \cong \mathscr{M}_{n}\left(\mathbb{C}\left[W_{I^{\prime}}\right]\right)$.

\section{Solomon Algebras and RenNer monoids}

Let $G$ be a group of Lie type in the sense of Carter [1]. Actually it suffices to assume as in [1, Chapter 2] that $G$ admits a split $B N$-pair satisfying some 
commutator relations. If $P$ is a parabolic subgroup, then we let $R_{u}(P)$ denote its unipotent radical. By a (finite) monoid of Lie type on $G$, we mean a finite regular monoid $M$ with zero 0 and unit group $G$ such that $M$ is generated $E=E(M)$ and $G$, and

(1) For all $e \in E$,

$$
P=P(e)=\{x \in G \mid x e=e x e\}, \quad P^{-}=P^{-}(e)=\{x \in G \mid e x=e x e\}
$$

are opposite parabolic subgroups of $G$ and for all $u \in R_{u}(P), v \in R_{u}\left(P^{-}\right)$, ue $=e=e v$.

(2) For all $e, f \in E, e M=f M$ or $M e=M f$ implies $x^{-1} e x=f$ for some $x \in G$.

Monoids of Lie type were introduced by the author [16] with a different name (regular split monoids) as an abstraction of certain properties of linear algebraic monoids [14, 19, 22]. They occur as the fixed points of endomorphisms of reductive monoids [20, 23].

Let $M$ be a monoid of Lie type on $G$. Following Solomon [24, 25], we are interested in the Hecke algebra

$$
\mathfrak{S}=\mathfrak{S}(M)=\varepsilon \mathbb{C}_{0}[M] \varepsilon
$$

where as before $\varepsilon=(1 /|B|) \sum_{b \in B} b$. We begin by discussing the structure of $M$. $G$ has a two-sided action on $M$. The $\mathscr{J}$-classes of $M$ are exactly the $G \times G$ orbits. Moreover the partially ordered set $\mathscr{U}=\mathscr{U}(M)$ of $\mathscr{J}$-classes forms a lattice. There is a cross section of idempotents $e_{J}(J \in \mathscr{U})$ such that $J=G e_{J} G$ and for all $J_{1}, J_{2} \in \mathscr{U}, e_{J_{1}} e_{J_{2}}=e_{J_{2}} e_{J_{1}}=e_{J_{1} \wedge J_{2}} . \Lambda=\left\{e_{J} \mid J \in \mathscr{U}\right\}$ is a cross section lattice in the sense of [14]. Moreover $M=E(M) G$ and for all $J \in \mathscr{U}$,

$$
E(J)=\left\{x^{-1} e_{J} x \mid x \in G\right\} .
$$

There is a type map $\lambda: \mathscr{U} \rightarrow 2^{\Gamma}$ such that for all $J \in \mathscr{U}, P\left(e_{J}\right)=P_{\lambda(J)}$, $P^{-}\left(e_{J}\right)=P_{\lambda(J)}^{-}$. We write $P_{J}, P_{J}^{-}, L_{J}, U_{J}, U_{J}^{-}, W_{J}$ for $P_{\lambda(J)}, P_{\lambda(J)}^{-}, L_{\lambda(J)}$, $U_{\lambda(J)}, U_{\lambda(J)}^{-}, W_{\lambda(J)}$, respectively. We call $\lambda$ the type of $M$. The type map completely determines the system of idempotents of $M$. Monoids of Lie type are first of all classified according to their type. For $J \in \mathscr{U}$, let

$$
K_{J}=\left\{x \in G \mid x e_{J}=e_{J} x=e_{J}\right\}
$$

Then $K_{J} \triangleleft L_{J}$. Let

$$
\mathscr{K}=\mathscr{K}(M)=\left\{\left(J, K_{J}\right) \mid J \in \mathscr{U}\right\} .
$$

The type map $\lambda$ along with $\mathscr{K}(M)$, classifies a monoid of Lie type up to isomorphism [16, Theorem 3.8].

Renner [22] generalized the classical Bruhat decomposition for reductive groups to reductive monoids by replacing the Weyl group by a finite inverse monoid (i.e., for each element $a$, there is a unique $x$ with $a x a=a, x a x=x$ ), which we call the Renner monoid. This decomposition was generalized to a monoid $M$ of Lie type by the author [16] as follows. Let

$$
E_{0}=\left\{\sigma^{-1} e_{J} \sigma \mid J \in \mathscr{U}, \sigma \in W\right\} .
$$

This is the analogue of the set of diagonal idempotents. Then $\left\langle N, E_{0}\right\rangle$ is an inverse monoid. If $a, b \in\left\langle N, E_{0}\right\rangle$ define

$$
a \equiv b \quad \text { if } B a B=B b B .
$$


Then $\equiv$ is an idempotent separating congruence on $\left\langle N, E_{0}\right\rangle$. Let

$$
\mathfrak{R}=\mathfrak{R}(M)=\left\langle N, E_{0}\right\rangle / \equiv .
$$

Then the unit group of $\Re$ is $W, E(\Re)=E_{0}$, and

$$
M=\bigsqcup_{w \in \mathfrak{R}} B w B
$$

Let $J \in \mathscr{U}$. Then the $\mathscr{J}$-class of $e_{J}$ in $\mathfrak{R}$ is $J_{1}=W e_{J} W$. Let $I=\lambda(J)$. Then by $[16, \S 4]$, the local group in $\Re$ at $e_{J}$ is $W_{I^{\prime}}$ as in Corollary 3.4 and $n=\left|E\left(J_{1}\right)\right|=|W| /\left|W_{I}\right|$. So by [3, Theorem 5.19],

$$
\mathbb{C}_{0}\left[J_{1}^{0}\right] \cong \mathscr{M}_{n}\left(\mathbb{C}\left[W_{I^{\prime}}\right]\right) \text {. }
$$

By Corollary 3.4,

$$
\varepsilon \mathbb{C}_{0}\left[J^{0}\right] \varepsilon \cong \mathscr{M}_{n}\left(\mathbb{C}\left[W_{I^{\prime}}\right]\right)
$$

Now for a maximal chain of ideals of $M$, the Rees factor semigroups are exactly $J^{0}(J \in \mathscr{U})$. Accordingly $\mathbb{C}_{0}[M]$ has a chain of ideals with the corresponding quotient algebras being isomorphic to $\mathbb{C}_{0}\left[J^{0}\right](J \in \mathscr{U})$. Hence $\mathfrak{S}$ has a chain of ideals with the corresponding quotient algebras being as in (24). Similarly $\mathbb{C}_{0}[\Re]$ has a chain of ideals with the corresponding quotient algebras being as in (23). We have therefore proved

Theorem 4.1. Let $M$ be a monoid of Lie type. Then the Solomon algebra $\mathfrak{S}(M)$ is semisimple and isomorphic to $\mathbb{C}_{0}[\Re]$ where $\mathfrak{R}$ is the Renner monoid of $M$.

Remark 4.2. For $M=\mathscr{M}_{n}\left(\mathbb{F}_{q}\right)$, Solomon [24] proves that $\mathfrak{S}(M) \cong \mathbb{C}_{0}[\Re]$ by making use of the result of Okniński and the author [11] on the semisimplicity of $\mathbb{C}_{0}[M]$. In this case $\mathfrak{R}$ is the symmetric inverse semigroup, the complex characters of which were explicitly computed by Munn [10]. We remark that our proof of Theorem 4.1 does not depend on [11].

Remark 4.3. Solomon [25] has posed the problem of finding an explicit isomorphism between $\mathfrak{S}(M)$ and $\mathbb{C}_{0}[\mathfrak{R}]$. Theorem 3.1 and Corollary 3.4 give a solution to this problem locally. We feel that the complete (global) solution may not be very pleasant. The natural idempotent in $\mathfrak{S}(M)$ corresponding to $e_{J} \in \Lambda$ is

$$
e_{J} \varepsilon=\frac{1}{|B|} \sum_{b \in B} e_{J} b \in \mathfrak{S}(M) .
$$

However this correspondence does not extend to an isomorphism between $\mathbb{C}_{0}[\mathfrak{R}]$ and $\mathfrak{S}(M)$ even for $M=\mathscr{M}_{2}\left(\mathbb{F}_{q}\right)$.

Problem 4.4. Does the map from the cross section lattice $\Lambda$,

$$
e_{J} \rightarrow \frac{1}{|B|} \sum_{b \in B} e_{J} b
$$

have a natural extension to $E_{0}=E(\Re)$ ?

In the notation of Theorem 3.1, we can try for $I=\lambda(J), \sigma \in D_{I}^{-1}$,

$$
\sigma^{-1} e_{J} \sigma \rightarrow \sum_{\theta \in D_{I}^{-1}} A_{\theta, \sigma}\left(\widetilde{Q}_{\theta, \sigma}^{I}\right)=\sum_{\substack{\theta \in D_{I}^{-1} \\ \theta \leq \sigma}} A_{\theta, \sigma}\left(\tilde{Q}_{\theta, \sigma}^{I}\right)
$$




\section{REFERENCES}

1. R. W. Carter, Finite groups of Lie type: conjugacy classes and complex characters, Wiley, New York, 1985.

2. A. H. Clifford, Matrix representations of completely simple semigroups, Amer. J. Math. 64 (1942), 327-342.

3. A. H. Clifford and G. B. Preston, Algebraic theory of semigroups, vol. 1, Math. Surveys Monographs, no. 7, Amer. Math. Soc., Providence, RI, 1961.

4. V. Deodhar, On some geometric aspects of Bruhat orderings. I: A finer decomposition of Bruhat cells, Invent. Math. 79 (1985), 499-511.

5. __ On some geometric aspects of Bruhat orderings. II: The parabolic analogue of KazhdanLusztig polynomials, J. Algebra 111 (1987), 483-506.

6. J. A. Green, On the structure of semigroups, Ann. of Math. (2) 54 (1951), 163-172.

7. N. Iwahori, On the structure of a Hecke ring of a Chevalley group over a finite field, J. Fac. Sci. Univ. Tokyo Sect. IA Math. 10 (1964), 215-236.

8. D. Kazhdan and G. Lusztig, Representations of Coxeter groups and Hecke algebras, Invent. Math. 53 (1979), 165-184.

9. W. D. Munn, On semigroup algebras, Proc. Cambridge Philos. Soc. 51 (1955), 1-15.

10. _ The characters of the symmetric inverse semigroup, Proc. Cambridge Philos. Soc. 53 (1957), 13-18.

11. J. Okniński and M. S. Putcha, Complex representations of matrix semigroups, Trans. Amer. Math. Soc. 323 (1991), 563-581.

12. __ Parabolic subgroups and cuspidal representations of finite monoids, Internat. J. Algebra Comput. 1 (1991), 33-47.

13. J. S. Ponizovskii, On matrix representations of associative systems, Mat. Sb. 38 (1956), 241-260.

14. M. S. Putcha, A semigroup approach to linear algebraic groups, J. Algebra 80 (1983), 164185.

15. __ Determinant functions on algebraic monoids, Comm. Algebra 11 (1983), 695-710.

16. __, Monoids on groups with BN-pairs, J. Algebra 120 (1989), 139-169.

17. __ Monoids of Lie type and group representations, Monoids and Semigroups with Applications (J. Rhodes, ed.), World Sci. Publ., Singapore and Teaneck, NJ, 1991, pp. 288-305.

18. _ Classification of monoids of Lie type, J. Algebra (to appear).

19. M. S. Putcha and L. E. Renner, The system of idempotents and the lattice of $\mathscr{J}$-classes of reductive algebraic monoids, J. Algebra 116 (1988), 385-399.

20. _ The canonical compactification of a finite group of Lie type, Trans. Amer. Math. Soc. 337 (1993), 305-319.

21. D. Rees, On semigroups, Proc. Cambridge Philos. Soc. 36 (1940), 387-400.

22. L. E. Renner, Analogue of the Bruhat decomposition for algebraic monoids, J. Algebra 101 (1986), 303-338.

23. __ Finite monoids of Lie type, Monoids and Semigroups with Applications (J. Rhodes, ed.), World Sci. Publ., Singapore and Teaneck, NJ, 1991, pp. 278-287.

24. L. Solomon, The Bruhat decomposition, Tits system and Iwahori ring for the monoid of matrices over a finite field, Geom. Dedicata 36 (1990), 15-49.

25. __ Reductive monoids, Notes of a talk given at MSRI Workshop on Representations of Reductive Groups over Finite Fields, Berkeley, November 1990.

Department of Mathematics, North Carolina State University, Raleigh, North CarOLINA 27695-8205

E-mail address: putcha@math.ncsu.edu 